\title{
Update sulla calcolosi renale
}

\author{
Martino Marangella, Cristiana Bagnis, Francesca Bermond, Silvia Berutti, Laura Fabbrini, \\ Paolo Gabella, Cristina Marcuccio, Giorgio Soragna, Alberto Tricerri, Corrado Vitale
}

\author{
S.C. Nefrologia e Dialisi, A.O. Ordine Mauriziano di Torino, Torino
}

\begin{abstract}
UPDATE ON NePhrolithiasis
Abstract. Many recent papers analyze the association between renal stone disease and other diseases that are typical of industrialized countries. Epidemiology studies from large series indicate that the prevalence of nephrolithiasis is higher among patients with metabolic syndrome, diabetes, and hypertension. Patients with nephrolithiasis also have an increased risk of myocardial infarction and stroke. It has been hypothesized that the common underlying defect could be insulin resistance. This, in turn, alters the urine biochemistry (i.e. more acidic pH and less urine citrate) thereby increasing the propensity of stone forming. In the diabetic rat renal steatosis has been implicated in the reduced production of ammonia, which has been shown to be reversible after PPAR $\gamma$ administration. Furthermore, pioglitazone was shown to be effective in reducing ethylene glycol-induced renal injury.

Another significant association concerns gout. Two recent papers report that both calcium and uric acid stone disease are more prevalent among patients with gout. The metabolic derangements found in gouty and non-gouty patients were quite similar. CT imaging in patients with gout indicates that the incidence of nephrolithiasis is underestimated if only based on stone history. Finally, stone episodes may occur many years before the first gouty attack.

Another interesting issue is that of a potential adverse effect of calcium and vitamin $D$ supplementation on the risk of stone formation. It has been shown that treated post-menopausal women have a slight but significantly higher risk of forming stones, independently of other interfering variables. From this the recommendation to evaluate the actual benefit of supplementation, even more in the face of its ineffectiveness to prevent fractures in older women.

Bariatric surgery is increasingly proposed for managing severe obesity, and in the last few years it has shown a widespread use in the US. Previous procedures of digestive diversion were often complicated by hyperoxaluria and renal oxalosis. More recent techniques, including Roux-en-Y gastric bypass and gastric bending, seem to give better outcomes. Hyperoxaluria and hypocitraturia following these procedures induce only minor increases in the risk of forming stones, but some recent reports of renal oxalosis after gastric bypass deserve attention.
\end{abstract}

Key words: Nephrolithiasis, Gout, Metabolic syndrome, Bariatric surgery

Conflict of interest: None.

Financial support: None.

Ricevuto: 11 Dicembre 2013; Accettato: 13 Dicembre 2013

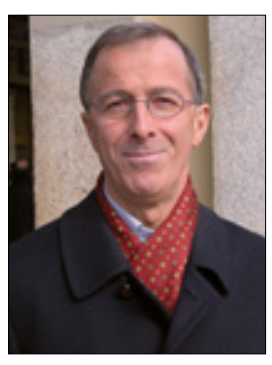

Martino Marangella

\section{Introduzione}

La calcolosi renale è, a tutt'oggi, oggetto di molte ricerche centrate su diversi aspetti, incluse patogenesi, eziologia, epidemiologia e relazione con altre condizioni morbose spesso associate. Pertanto, risulta abbastanza utile una rassegna di alcune delle novità sul tema, con spunti interessanti e fonti di ulteriori ricerche. A questo, purtroppo, non corrisponde un avanzamento significativo nell'approccio terapeutico e di prevenzione della calcolosi, in particolare della sua forma idiopatica, che è anche la più frequente.
In questo articolo riprenderemo alcuni dei temi riportati nella letteratura recente e presentati nel Simposio del GdS Metabolismo Minerale ed Elementi in Traccia tenutosi a Milano il 15 Dicembre del 2012.

\section{Calcolosi renale e sindrome metabolica, obesità e diabete mellito}

Molta recente letteratura si è occupata della relazione fra nefrolitiasi idiopatica, patologie metaboliche e obesità. Si tratta, in tutti i casi, di patologie di elevato impatto epidemiologico e questo rende difficoltoso stabilire un'associazione significativa fra le stesse. Da molti studi epidemiologici, sembra, tuttavia, emergere una relazione fra calcolosi renale, diabete, sindrome metabolica e obesità. Queste aumentano il rischio di patologia 
cardio-vascolare e, di conseguenza, si ha un aumento del rischio di infarto e di ictus rilevato anche nella popolazione litiasica.

Rendina et al. $(1,2)$ hanno confrontato il profilo clinico e metabolico di pazienti con calcoli renali di calcio ossalato senza e con sindrome metabolica (SM) associata versus controlli sani. Dal punto di vista biochimico, i calcolotici, sia con che senza $\mathrm{SM}$, avevano un maggior rischio, espresso come odds ratio, di ipocitraturia, iperossaluria e ipercalciuria. Per contro, la sodiuria era più elevata solo in quelli con SM. La sodiuria si correlava con la saturazione (activity product ratio) rispetto al calcio ossalato, con una pendenza maggiore per i pazienti con SM. Il rischio litogeno si riduceva in modo significativo solo nei pazienti aderenti alla dieta iposodica.

In precedenti lavori era stato trovato che il rischio di calcolosi renale era correlato con il numero di segni della SM nella popolazione generale (3).

Dal momento che la SM predispone alla patologia cardio-vascolare, gli epidemiologi hanno studiato il rapporto fra quest'ultima e la calcolosi renale. Ne sono risultati dati interessanti.

Il rischio di infarto miocardico, espresso come hazard ratio, era aumentato del $43 \%$ in un follow-up medio di 9 anni fra 4.564 pazienti con anamnesi di calcolosi renale confrontati con 10.860 soggetti sani. L'aumento rimaneva significativo al 31\% anche dopo l'aggiustamento per altre comorbilità (4).

In un lavoro analogo è stato visto che la prevalenza di ictus, in un follow-up di 5 anni, in 25.181 pazienti litiasici, era pari a 1.78/100 pazienti/anno versus 1.25 fra 125.905 soggetti senza storia di calcolosi. Anche in questa vasta coorte, il rischio (hazard ratio) di ictus era pari a $1.43(\mathrm{p}<0.001)$ anche dopo l'aggiustamento per ipertensione, diabete, iperdislipidemia, patologia cardio-vascolare, gotta e obesità (5). Da interpretare il reperto di valori di HR maggiori per i pazienti con calcolosi del rene, rispetto a quelli con calcolosi ureterale (HR 1.73 versus $1.34 \mathrm{e}$, dopo aggiustamento, 1.5 versus 1.42 ).

Un altro studio epidemiologico ha riportato un rischio maggiore di sviluppo di diabete mellito dopo un follow-up di 5 anni in soggetti litiasici, con valori di HR di 1.48 e 1.32 dopo l'aggiustamento (6).

I dati epidemiologici di associazione fra patologie molto diffuse nelle società industrializzate sono, almeno in parte, riconducibili a fattori di rischio comuni, come, per esempio, le abitudini dietetiche dei paesi ricchi, in cui il consumo di proteine di origine animale, di sale e di zuccheri semplici e, in particolare, di fruttosio è più elevato.

Un aspetto particolare di questa associazione è schematizzato nella Figura 1. Un fattore patogenetico comune può essere la resistenza all'azione dell'insulina. Lo stato di cronico iperinsulinismo si associa a diabete mellito e alla sindrome metabolica, mentre l'insulina di per sé esercita, a livello renale, un'azione di inibizione dell'ammoniogenesi e, di conseguenza, la produzione di urine più acide che, è noto, aumentano il rischio di calcolosi urica $(7,8)$. Insieme all'emissione di urine acide è stata osservata una ridotta escrezione di citrato nei ratti geneticamente modificati con DM tipo 2 e sindrome metabolica (9). Il trattamento con pioglitazone ha indotto un aumento dose-dipendente dell'escrezione di citrato (9). Nei ratti diabetici è stato confermato il difetto di ammoniogenesi renale, che è stato attribuito a un accumulo di trigliceridi a livello tubulo-interstiziale (10). Il

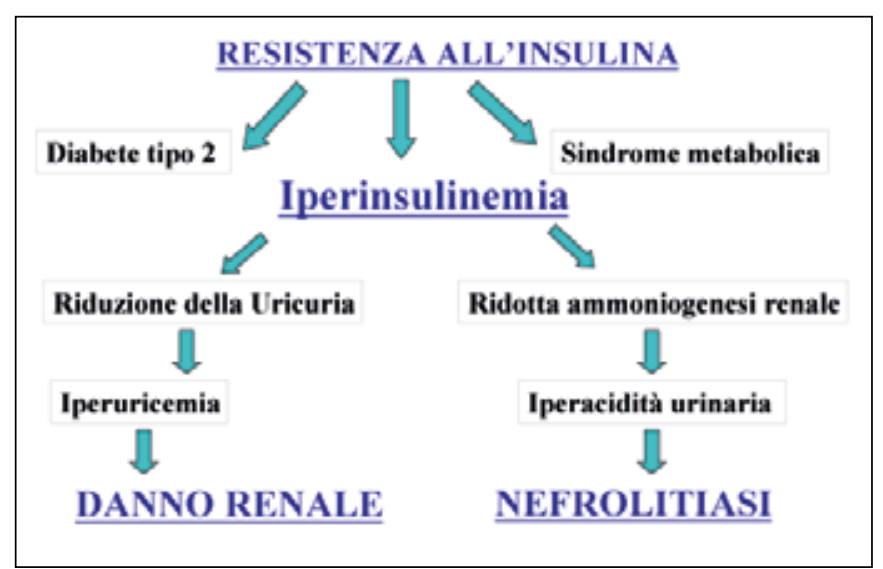

Fig. 1 - Ipotesi del ruolo della resistenza all'insulina nella patogenesi della nefrolitiasi e dell'iperuricemia.

difetto è stato corretto dalla terapia con tiazolidinedioni, agonisti del PPAR $\gamma$, che hanno ridotto il grado di steatosi renale (10). Il pioglitazone è stato anche sperimentato nella prevenzione del danno renale indotto da etilen glicole (EG). Infatti, in un modello sperimentale su ratto, la somministrazione di questo agonista del recettore PPAR $\gamma$ ha ridotto la deposizione di cristalli di calcio ossalato a livello tubulo-interstiziale e i fenomeni di apoptosi, di perossidazione e di infiammazione, conseguenti all'esposizione all'EG. L'effetto era indipendente da modificazioni dell'escrezione di fattori di rischio litogeno (11).

Questa serie di lavori propone ipotesi patogenetiche volte a chiarire la relazione epidemiologica fra calcolosi renale e DM e SM. La resistenza all'azione dell'insulina sembra esserne il denominatore comune, facendo emergere una serie di effetti che inducono un aumento del rischio di calcolosi non solo urica (urine più acide) ma anche di calcio ossalato (ipocitraturia). L'uso di farmaci antidiabetici ha attenuato il rischio litogeno in modelli sperimentali sul ratto. Non si deve, con questo, dedurre che i pazienti litiasici debbano essere trattati con agonisti del $\operatorname{PPAR} \gamma$, ma questi dati rinforzano gli aspetti patogenetici del rapporto fra calcolosi e SM e DM.

\section{Gotta e calcolosi renale}

È noto da anni che i pazienti gottosi hanno una prevalenza di calcolosi maggiore rispetto ai controlli. La calcolosi non è necessariamente urica, ma è stata descritta anche una quota significativa di calcolosi calcica (12). Una recente indagine ha esaminato la composizione dei calcoli in pazienti gottosi e ha valutato l'incidenza delle anomalie metaboliche note come fattori di rischio di calcolosi calcica e urica (13). Sono stati confrontati 181 pazienti gottosi con altrettanti litiasici non gottosi, confrontabili per sesso, età e indice di massa corporea. La distribuzione percentuale per tipo di calcolosi è riportata nella Tabella I. Si noti che, fra i gottosi, l'incidenza di calcolosi urica è quasi pareggiata da quella di calcolosi calcica. Non solo, ma nei pazienti gottosi in terapia cronica con allopurinolo la distribuzione percentuale di calcolosi diventa paragonabile con quella dei non gottosi, con calcoli di acido urico nel $25.7 \%$ dei casi e di 
calcio ossalato nel 61.8\%. Gli Autori hanno anche valutato l'incidenza delle principali anomalie metaboliche e i risultati sono riportati nella Tabella II. Non è emersa alcuna differenza fra $\mathrm{i}$ due gruppi nei valori medi di escrezione dei fattori di rischio, mentre, inaspettatamente, l'iperossaluria era un po' più frequente fra i gottosi $(\mathrm{p}<0.01)$. Dai risultati di questo lavoro, gli Autori sottolineano la necessità di sottoporre a indagini metaboliche complete anche i pazienti gottosi, non dando per scontato che la loro calcolosi sia su base uratica.

Un altro interessante studio sul rapporto fra gotta e calcolosi renale ha valutato il rapporto fra anamnesi di calcolosi e presenza di calcoli alla TC. È così emerso che, a fronte di una diagnosi radiologica di calcolosi in 157/463 pazienti gottosi (34\%), solo in $75(16 \%)$ vi era una storia di calcolosi (14). Nei 157 pazienti con diagnosi di calcolosi alla $\mathrm{TC}$, la funzione renale era peggiore, rispetto ai non calcolotici, e il pH urinario era più acido. I pazienti con calcolosi bilaterale avevano GFR inferiore e $\mathrm{pH}$ urinario più acido rispetto a quelli con calcolosi monolaterale. La composizione dei calcoli è stata dedotta dalla misura della densità in Unità Hounsfield, dimostrando che solo $1 / 3$ dei pazienti gottosi aveva calcoli di acido urico. Infine, nel 24\% dei pazienti con anamnesi positiva, l'episodio di calcolosi aveva preceduto il primo attacco di gotta. L'ovvia conclusione degli Autori è che l'incidenza della calcolosi renale nei pazienti con gotta è sottostimata e che la presenza di calcoli ha un impatto negativo sulla funzione renale dei pazienti.

TABELLA I - DISTRIBUZIONE PERCENTUALE DELLA CALCOLOSI RENALE PER COMPOSIZIONE DEI CALCOLI IN LITIASICI CON E SENZA GOTTA

\begin{tabular}{lcc}
\hline Composizione & Gottosi & Non gottosi \\
\hline Calcio ossalato monoidrato & 45.2 & $68.6^{*}$ \\
Calcio ossalato biidrato & 0.6 & 3.5 \\
Calcio fosfato & 1.6 & 4.9 \\
Acido urico & 52.2 & $22.3^{*}$ \\
Struvite & 0 & 0.3 \\
Misti & 20.7 & 27 \\
\hline
\end{tabular}

$* \mathrm{p}<0.001$

TABELLA II - DISTRIBUZIONE PERCENTUALE DELLE PRINCIPALI ANOMALIE METABOLICHE IN LITIASICI CON E SENZA GOTTA

\begin{tabular}{lcc}
\hline Anomalia metabolica & Gottosi & Non gottosi \\
\hline Ipercalciuria & 28 & 32 \\
Iperossaluria & 74 & $61^{*}$ \\
Ipocitraturia & 58 & 49 \\
Iperuricuria & 19 & 14 \\
Urine a pH acido & 49 & 39 \\
\hline
\end{tabular}

$* \mathrm{p}<0.01$

\section{Calcolosi renale, dieta, calcio e vitamina $D$}

Un argomento molto trattato in letteratura concerne i possibili effetti negativi sulla calcolosi renale dei supplementi di calcio e vitamina $\mathrm{D}$, largamente prescritti nelle donne in menopausa. Non vi è, a oggi, uniformità di vedute in merito.

Una pubblicazione recente rivaluta i risultati della Women's Health Initiative (WHI), un RCT di supplementazione di calcio e vitamina D condotto negli Stati Uniti. Lo studio ha confrontato 36.282 donne fra i 50 e i 79 anni, poste in terapia con $500 \mathrm{mg}$ di calcio carbonato e 200 UI di vitamina D, per due volte al giorno, con 18.176 in trattamento con placebo. Dopo un followup medio di 7 anni si sono registrati 449 casi di calcolosi fra il gruppo calcio e vitamina D versus 381 nel gruppo placebo, con un aumento del rischio relativo pari al 17\% (15). Nessuna delle covariabili prese in considerazione (razza, grado di istruzione, BMI, fumo, alcol, ipertensione, diabete mellito) ha modificato il livello di rischio. Ugualmente ininfluenti sono risultati l'apporto dietetico di calcio o l'uso di supplementi di calcio prima dell'avvio del trial. Pur ammettendo i limiti di uno studio basato sull'autodiagnosi di calcolosi, i dati invitano a non sottovalutare l'impatto di calcio e vitamina D sul rischio di calcolosi, e questo anche alla luce di indagini recenti da cui sembra emergere che $\mathrm{i}$ supplementi di calcio e vitamina D non hanno un effetto significativo, specialmente nelle donne anziane, nella prevenzione delle fratture ossee (16).

Il probabile meccanismo attraverso cui la vitamina $\mathrm{D}$ può aumentare il rischio di calcolosi è quello dell'induzione di ipercalciuria. In uno studio recente, 29 pazienti con anamnesi di calcolosi e con livelli basali di $25(\mathrm{OH}) \mathrm{D}_{3}<30 \mathrm{ng} / \mathrm{mL}$ venivano trattati con $50.000 \mathrm{UI} /$ settimana di ergocalciferolo per un periodo di 8 settimane. A fronte di un aumento significativo dei livelli di $25(\mathrm{OH}) \mathrm{D}_{3}$ da 17.6 a $35.6 \mathrm{ng} / \mathrm{mL}$, non si sono avute variazioni significative dell'escrezione calcica, anche se, in 11 pazienti, la calciuria è aumentata di $20 \mathrm{mg} / \mathrm{dL}$, parallelamente a un aumento della sodiuria (17). La conclusione dello studio è che una cauta replezione dei livelli di vitamina $\mathrm{D}$ anche in pazienti con calcolosi renale non comporta un aumento significativo del rischio litogeno.

Ugualmente ininfluente era, sul rischio litogeno, espresso come Indice di Tiselius, un elevato apporto di ossalato prescritto in una dieta controllata a volontari sani, a patto che l'apporto dietetico di calcio fosse adeguato (18).

\section{Calcolosi renale e chirurgia bariatrica}

L'enorme aumento della prevalenza di grandi obesi nei paesi industrializzati ha avuto come conseguenza la larga diffusione di interventi di chirurgia bariatrica volti a ridurre in modo significativo l'assorbimento intestinale di nutrienti. Negli Stati Uniti, è stato registrato un incremento della chirurgia bariatrica da 13.365 interventi nel 1998 a 72.177 nel 2002, con una proiezione al 2003 di 102.794 interventi, di cui 1' $80 \%$ rappresentato da bypass gastrici (19). In anni più recenti è stata introdotta la tecnica del bendaggio gastrico in alternativa a quella cosiddetta di ansa a Y alla Roux (Fig. 2).

In uno studio del 2010 del gruppo di Dallas erano stati confrontati 19 soggetti obesi sottoposti a intervento di bypass gastrico 


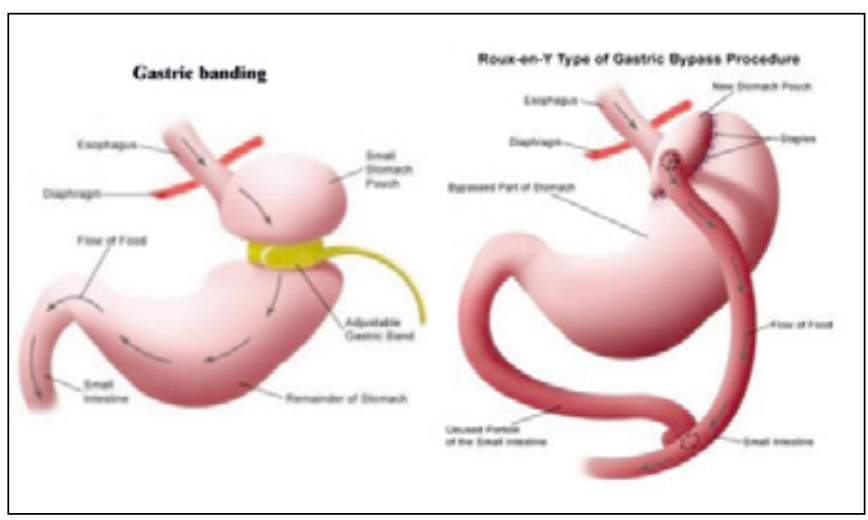

Fig. 2 - Interventi di chirurgia bariatrica. A sinistra bendaggio gastrico. A destra bypass gastrico con ansa a Y alla Roux.

con 19 obesi, confrontabili per sesso, età e BMI. La biochimica urinaria aveva mostrato un aumento dell'escrezione dell'ossalato e una riduzione di quella di citrato, con una prevalenza di iperossaluria del $47 \%$ versus il $10.5 \%$ nei due gruppi e di ipocitraturia del $63 \%$ versus il $5 \%(\mathrm{p}<0.01)$. Tuttavia, la contemporanea riduzione della calciuria, conseguente al malassorbimento di calcio, attenuava gli effetti negativi sullo stato di saturazione rispetto al calcio ossalato (20).

Le anomalie di escrezione dell'ossalato conseguenti al bypass gastrico non sono state osservate nei pazienti sottoposti a bendaggio gastrico. In 18 pazienti sottoposti a gastric bending, l'escrezione di ossalato è risultata paragonabile a quella dei soggetti normali e dei litiasici recidivanti, ma inferiore rispetto a quella dei soggetti con ansa alla Roux $(35.4 \pm 3.1$ versus $60.8 \pm$ $3.5 \mathrm{mg} / 24 \mathrm{~h}, \mathrm{p}<0.01)(21)$.

Da questi lavori si desume che il rischio di calcolosi renale, conseguente all'iperossaluria, molto frequente negli interventi di derivazione bilio-digestiva utilizzati in passato per il trattamento dell'obesità, è minore, a condizione che il paziente mantenga sempre un corretto stato di idratazione. Sono stati, infatti, descritti in letteratura casi di nefropatia ossalotica con insufficienza renale severa, anche irreversibile, in pazienti portatori di ansa alla Roux (22). È stato anche riportato che la supplementazione di citrato di potassio è in grado di attenuare sia il rischio di calcolosi renale che quello di perdita della massa ossea conseguenti alla chirurgia bariatrica (23).

Accanto a questi effetti potenzialmente sfavorevoli, gli interventi di chirurgia bariatrica hanno, comunque, un potenziale impatto positivo sulla funzione renale nei pazienti obesi. Infatti, la chirurgia migliora la tolleranza glucidica riducendo la resistenza all'insulina, migliora il profilo lipidico, attenua l'ipertensione arteriosa e, da ultimo, riduce morbilità e mortalità dei pazienti (24).

In generale, l'impatto della calcolosi sulla funzione renale non sembra trascurabile. Infatti, nel grosso studio epidemiologico condotto su una grande coorte di pazienti in Canada (Alberta Kidney Disease Network), è stato visto che l'anamnesi di calcolosi renale raddoppiava il rischio di insufficienza renale cronica anche end-stage, con un andamento indipendente dal sesso e dall'età. Nei pazienti con un singolo episodio, il rischio relativo di IRC end-stage era pari a 2.36, con un aumento a 2.82 nei pazienti con almeno un secondo episodio (25).

\section{Conclusioni}

In questo up-to-date sulla calcolosi renale sono stati esposti i risultati di lavori recenti aventi come oggetto il rapporto fra nefrolitiasi e anomalie metaboliche. Se questo rapporto sia casuale o causale non è chiaro, a oggi, e la grande frequenza delle due patologie non consente di stabilire se l'associazione abbia una robusta significatività statistica. Resta il dato sperimentale che collega l'aumentata resistenza all'azione dell'insulina ad alterazioni del metabolismo renale e, di conseguenza, ad anomalie dei fattori di rischio urinario.

L'altro aspetto importante, e, in qualche modo, collegato al primo, è quello della chirurgia bariatrica come procedura con un potenziale litogeno significativo. Pur non avendo la frequenza e la severità di complicanze litiasiche tipiche dei vecchi interventi di derivazione digestiva, le procedure moderne richiedono un'attenta sorveglianza dei pazienti che vi si sottopongono e il problema è potenzialmente aumentato dalla sempre maggiore diffusione delle stesse.

\section{Riassunto}

Molti lavori recenti analizzano l'associazione fra calcolosi renale e altre patologie non trasmissibili tipiche dei paesi industrializzati. I dati epidemiologici, su casistiche ampie, indicano un aumento di incidenza della calcolosi in pazienti con sindrome metabolica, obesità e ipertensione. Viene anche descritto un aumento del rischio di infarto miocardico e di ictus nei litiasici. Si ipotizza che il denominatore comune in queste patologie sia l'aumento della resistenza all'insulina. Questo causa alterazioni della biochimica urinaria, $\mathrm{pH}$ più acido e riduzione della citraturia, tali da aumentare il rischio litogeno. Nel ratto diabetico è stata descritta una steatosi renale che riduce l'ammoniogenesi e che è reversibile con una terapia con PPAR $\gamma$. Il pioglitazone è stato efficace nel ridurre il danno renale indotto nel ratto da etilen glicole. Altra associazione nota è quella fra calcolosi renale e gotta. Due recenti studi documentano un'incidenza della calcolosi calcica e non solo urica nella gotta, con anomalie metaboliche in parte simili a quelle dei pazienti non gottosi. L'indagine TC mostra che l'incidenza di calcolosi è sottostimata nella gotta e, inoltre, la calcolosi, in alcuni pazienti, precede anche di molti anni l'attacco gottoso.

Un altro argomento analizza il potenziale effetto che favorisce la calcolosi nelle donne trattate con calcio e vitamina D. Emergono un modesto ma significativo aumento del rischio litogeno indipendente da altre covariabili e un conseguente invito all'attenta valutazione del rapporto rischio/beneficio. La chirurgia bariatrica per la correzione della grave obesità era, in passato, gravata da un elevato rischio di calcolosi renale iperossalurica con quadri anche di ossalosi severa. Negli ultimi anni si sono diffuse tecniche meno litogene come il bendaggio gastrico e il bypass gastrico alla Roux. Iperossaluria e ipocitraturia conseguono a questi interventi e il rischio 
di calcolosi è di gran lunga inferiore, ma restano segnalazioni in letteratura di casi di ossalosi renale specialmente dopo bypass gastrico alla Roux.

Parole chiave: Nefrolitiasi, Gotta, Sindrome metabolica, Chirurgia bariatrica

Dichiarazione di conflitto di interessi: Gli Autori dichiarano di non avere conflitto di interesse.

Contributi economici agli Autori: Gli Autori dichiarano di non aver ricevuto sponsorizzazioni economiche per la preparazione dell'articolo.
Indirizzo degli Autori:

Dr. Martino Marangella

S.C. Nefrologia e Dialisi

A.O. Ordine Mauriziano di Torino

Largo Turati 62

10128 Torino

mmarangella@mauriziano.it

\section{Bibliografia}

1. Rendina D, Mossetti G, De Filippo G, et al. Association between metabolic syndrome and nephrolithiasis in an inpatient population in southern Italy: role of gender, hypertension and obesity. Nephrol Dial Transplant 2009; 24: 900-6.

2. Rendina D, De Filippo G, Zampa G, Muscariello R, Mossetti G, Strazzullo P. Characteristic clinical and biochemical profile of recurrent calcium-oxalate nephrolithiasis in patients with metabolic syndrome. Nephrol Dial Transplant 2011; 26: 2256-63.

3. West B, Luke A, Durazo-Arvizu RA, et al. Metabolic syndrome and self-reported history of kidney stones: the National Health and Nutrition Examination Survey (NHANES III) 1988-1994. Am J Kidney Dis 2008; 51: 741-7.

4. Rule AD, Roger VL, Melton LJ, et al. Kidney Stones Associate with Increased Risk for Myocardial Infarction. J Am Soc Nephrol 2010; 21: 1641-4.

5. Chung SD, Liu SP, Keller JJ, Lin HC. Urinary calculi and an increased risk of stroke: a population-based follow-up study. $\mathrm{Br}$ J Urol Int 2012; 110: E1053-9.

6. Chung SD, Chen YK, Lin HC. Increased Risk of Diabetes in Patients With Urinary Calculi: A 5-Year Followup Study. J Urol 2011; 186: 1888-93.

7. Sakhaee K, Adams-Huet B, Moe OW, Pak CY. Pathophysiologic basis for normouricosuric uric acid nephrolithiasis. Kidney Int 2002; 62: 971-9.

8. Sakhaee K, Maalouf NM, Sinnott B. Kidney Stones 2012: Pathogenesis, Diagnosis and Management. J Clin Endocrinol Metab 2012; 97: 1847-60.

9. Ina A, Kohjimoto Y, Mori T, Kuramoto T. Insulin resistance increases the risk of urinary stone formation in rat model of metabolic syndrome. BJU Int 2010; 106: 1550-4.

10. Bobulescu IA, Dubree M, Zhang J, et al. Reduction of renal triglyceride accumulation: effects on proximal tubule $\mathrm{Na}+\mathrm{H}+$ exchange and urinary acidification. Am J Physiol Renal Physiol 2009; 297: F1419-26.

11. Taguchi K, Okada A, Yasui T. Pioglitazone, a peroxisome proliferator pctivated receptor agonist, decreases renal crystal deposition, oxidative stress and inflammation in hyperoxaluric rats. $\mathrm{J}$ Urol 2012; 188: 1002-11.
12. Kramer HM, Curhan G. The association between gout and nephrolithiasis: the National Health and Nutrition Examination Survey III, 1988-1994. Am J Kidney Dis 2002; 40: 37-42.

13. Marchini GS, Sarkissian C, Tian D, Gebreselassie S, Manoj Monga M. Gout, stone composition and urinary stone risk: a case matched comparative study. J Urol 2013; 189: 1334-9.

14. Shimizu T, Kitada H, Umeyama M, et al. Novel Evaluation of Nephrolithiasis as a Complication of Gout: A Cross-Sectional Study Using Helical Computerized Tomography. J Urol 2013; 189: 1747-52.

15. Wallace RB, Wactawski-Wende J, O'Sullivan MJ, et al. Urinary tract stone occurrence in the Women's Health Initiative (WHI) randomized clinical trial of calcium and vitamin D supplements. Am J Clin Nutr 2011; 94: 270-7.

16. Kuehn BM. USPSTF: Taking Vitamin D and Calcium doesn't prevent fractures in older women. JAMA 2012; 308: 225-6.

17. Leaf DE, Korets R, Taylor EN, et al. Effect of Vitamin D Repletion on Urinary Calcium Excretion among Kidney Stone Formers. Clin J Am Soc Nephrol 2012; 7: 829-34.

18. Lange JN, Wood KD, Mufarrij MF, et al. Impact of dietary calcium and oxalate ratios on stone risk. Urology 2012; 79: 1226-9.

19. Heena P. Santry HP, Gillen DL, Lauderdale DS. Trends in bariatric surgical procedures. JAMA 2005; 294: 1909-17.

20. Maalouf NM, Tondapu P, Guth ES, et al. Hypocitraturia and hyperoxaluria after Roux-en-Y gastric bypass surgery. J Urol 2010; 183: 1026-30.

21. Semins MJ, Asplin JR, Steele K, et al. Effect of restrictive bariatric surgery on urinary stone risk factors. Urology 2010; 76: 826-9.

22. Nasr SH, D'Agati VD, Said SM, et al. Oxalate nephropathy complicating Roux-en-Y gastric bypass: an underrecognized cause of irreversibile renal failure. Clin J Am Soc Nephrol 2008; 3: $1676-83$.

23. Sakhaee K, Griffith C, Pak CY. Biochemical control of bone loss and stone-forming propensity by potassium citrate after bariatric surgery. Surg Obes Rel Dis 2012; 8: 67-72.

24. Ahmed MH, Byrne CD. Bariatric surgery and renal function: a precarious balance between benefit and harm. Nephrol Dial Transplant 2010; 25: 3142-7.

25. Alexander RT, Hemmelgarn BR, Wiebe N, et al. Kidney stones and kidney function loss: a cohort study. BMJ 2012; 345: e5287. 